\title{
Isolated effect of maternal thyroid-stimulating hormone, free thyroxine and antithyroid peroxidase antibodies in early pregnancy on gestational diabetes mellitus: a birth cohort study in China
}

\author{
Kun Huang ${ }^{1), 2)}$, Yeqing $\mathrm{Xu}^{3)}$, Shuangqin $\mathrm{Yan}^{3)}$, Tao $\mathrm{Li}^{4)}$, Yuanhong $\mathrm{Xu}^{4)}$, Peng $\mathrm{Zhu}^{1), 2)}$ and Fangbiao Tao ${ }^{1), 2)}$ \\ 1) School of Public Health, Anhui Medical University, Hefei, Anhui 230032, P.R. China \\ 2) Anhui Provincial Key Laboratory of Population Health and Aristogenics, Hefei, Anhui 230032, P.R. China \\ 3) Maternal and Child Health Center, Ma'anshan, Anhui 243011, P.R. China \\ 4) Department of Clinical Laboratory, The First Affiliated Hospital of Anhui Medical University, Hefei, Anhui 230082, P.R. China
}

\begin{abstract}
This article aims to understand the isolated effect of maternal thyroid-stimulating hormone (TSH), free thyroxine $\left(\mathrm{FT}_{4}\right)$ and antithyroid peroxidase antibodies (TPOAb) in early pregnancy on gestational diabetes mellitus (GDM). Based on a birth cohort, pregnant women presented to maternity hospitals for the first antenatal care from Nov 2008 to Oct 2010 were invited to participate in the study. A self-administered questionnaire was asked to complete to collect data on socio-economic variables, previous adverse pregnancy outcomes, method of conception, previous endocrinic and metabolic diseases, and pregnancy-related anxiety in $1^{\text {st }}$ trimester of the index pregnancy. Pre-pregnancy BMI was measured. Serum samples were collected, and TSH, $\mathrm{FT}_{4}$ and TPOAb were assayed. GDM was confirmed from medical records screened on 24-28 gestational weeks by using oral glucose tolerance test (OGTT). The prevalence of isolated subclinical hypothyroidism, hypothyroidemia and positive TPOAb in early pregnancy was $2.0 \%, 2.0 \%$ and $12.8 \%$. Prevalence of GDM in women with the isolated subclinical hypothyroidism, hypothyroxinemia and positive TPOAb was $2.9 \%, 2.8 \%$ and $3.1 \%$, respectively, which were all higher than that detected in euthyroidism women (1.2\%). Women with isolated positive TPOAb had significantly higher TSH and lower $\mathrm{FT}_{4}$ level compared with euthyroidism women. It was found that isolated positive TPOAb in early pregnancy increased the risk of GDM, adjusted RR and 95\%CI being 2.541(1.037-6.226). No significant relationships were identified between isolated sub-clinical hypothyroidism or hypothyroxinemia with GDM. In conclusion, isolated thyroid autoimmunity, represented by positive TPOAb, in early pregnancy were associated with GDM independent of TSH and $\mathrm{FT}_{4}$.
\end{abstract}

Key words: Thyroid-stimulating hormone, Free thyroxine, Antithyroid peroxidase antibodies, Gestational diabetes mellitus, Early pregnancy

GESTATIONAL DIABETES MELLITUS (GDM) is defined as glucose intolerance first identified during pregnancy. It is one of the most common pregnancy complications. Documented GDM prevalence varies from $1 \%$ and $14 \%$ [1]. The reported rate in China is between $1 \%$ and $5 \%$, and it has demonstrated a marked and progressive rise during the recent years [2].

Women with GDM are usually characterized with pregnancy-induced insulin resistance. Most of them can return to normal glucose tolerance after childbirth, while some women have an increased risk of overt diabetes in

Submitted Aug. 23, 2018; Accepted Dec. 23, 2018 as EJ18-0340 Released online in J-STAGE as advance publication Jan. 24, 2019 Correspondence to: Fangbiao Tao, School of Public Health, Anhui Medical University, No 81 Meishan Road, Hefei city, Anhui Province 230032, P.R. China.

E-mail: fangbiaotao@163.com the late life [3]. Thyroid hormones are important determinants of glucose homeostasis [4]. Several changes occur in maternal thyroid function during pregnancy, and failure to adapt to these physiological changes will result in thyroid dysfunction [5]. Thyroid dysfunction and/or positive antibodies are relatively common during pregnancy $[6,7]$, and may have profound effects on glucose metabolism. Studies suggest that GDM occurred more often in women with overt hypothyroidism. It is now well established that not only overt, but mild thyroid dysfunction also has adverse effects on maternal outcome. The findings are controversial. Sub-clinical hypothyroidism was reported to be related with diabetes diagnosed during pregnancy [8]. Karakosta PD reported that subclinical hypothyroidism with positive autoantibodies was associated with GDM, while that with negative autoantibodies was not found to be related with GDM [9]. 
Domestic researchers revealed that serum free thyroxine $\left(\mathrm{FT}_{4}\right)$ levels were lower in GDM women compared with non-GDM women $[10,11]$, but Shahbazian H et al. did not observe any differences in thyroid function between GDM women and controls [12]. Apart from the differences in GDM diagnostic criteria used in various studies, another important reason for the above-mentioned disputes is whether to consider the thyroid autoimmune status. GDM is associated with many non-organ-specific auto-antibodies. Thyroid peroxidase (TPO) plays a key role in the synthesis and secretion of thyroid hormones. It is also major autoantigen in autoimmune thyroid diseases [13]. High titres of thyroid peroxidase antibody (TPOAb) in a GDM pregnancy could potentially compromise the known fetal and maternal complications associated with GDM [14]. Till now, isolated effect of $\mathrm{TSH}, \mathrm{FT}_{4}$ or positive TPOAb on the risk of GDM is unclear. Therefore, in the current study, based on a population-based birth cohort, we aim to understand the association of isolated subclinical hypothyroidism, hypothyroxinemia and positive TPOAb in early pregnancy with GDM, and assess the independent role of $\mathrm{TSH}, \mathrm{FT}_{4}$ and TPOAb on GDM occurrence.

\section{Materials and Methods}

This study was a part of broader project entitled "The China-Anhui birth cohort study (C-ABCS)". The cohort profile had been described elsewhere [15]. Ethical approval was obtained from the Bio-medical Ethical Committee of Anhui Medical University (approval no.2008020).

\section{Participants}

The participants of this study came from Ma'anshan city, Anhui province, China. Pregnant women presented to Maternal and Child Health Center in Ma'anshan city for the first antenatal care from Nov 2008 to Oct 2010 were invited to participate in the study. They were recruited into the $\mathrm{C}$-ABCS after informed consents were granted.

\section{Questionnaire survey}

Women were asked to complete a self-administered questionnaire to collect the following data: 1) Socioeconomic variables, including maternal age, maternal education level, residence and family income. 2) Previous adverse pregnancy outcomes, including spontaneous abortion, induced abortion, intrauterine fetal demise or stillbirth, extra-uterine pregnancy, malformation. Women who had any one of the above outcomes were defined as having previous adverse pregnancy outcomes. 3) Previous endocrinic and metabolic diseases and family histories, such as diabetes mellitus and thyroid diseases. 4)
Method of conception. Women were asked whether the index pregnancy was spontaneous or by use of assisted reproductive technologies (ART). 5) Pregnancy-specific anxiety during the $1^{\text {st }}$ trimester in the index pregnancy. It was evaluated by using self-designed pregnancy-specific anxiety questionnaire. The questionnaire consisted of three subscales: fear for women's own health, fear for baby's health and fear for childbirth. Women were asked to self-rate their anxiety status from 1-4 points varying from no worries, occasionally worried, often worried to always worried. Higher the total score was, higher level of anxiety the women had. The Cronbach's alpha coefficient and test-retest reliability coefficient were 0.81 and 0.79 , respectively $[16,17]$.

Body height and weight of pregnant women were measured and used to calculate the pre-pregnancy body mass index (BMI) with the formula weight $(\mathrm{kg}) /$ height $(\mathrm{m})^{2}$. Overweight and obesity was defined when BMI $\geq 24.0$ [18].

\section{Assessment of thyroid function}

Women's blood samples were collected during the first visit. Serum were preserved at $-80^{\circ} \mathrm{C}$ after centrifugation. Quantitative analyses of $\mathrm{TSH}, \mathrm{FT}_{4}$ and $\mathrm{TPOAb}$ were performed by chemiluminescent immunoassays on an automated platform (LIAISON analyzer; DiaSorin SpA., Saluggia, Italy) in the department of clinical laboratory in $1^{\text {st }}$ Affiliated Hospital of Anhui Medical University. The measured concentration of TSH was between $0.004 \mathrm{mIU} / \mathrm{L}$ and $100 \mathrm{mIU} / \mathrm{L}$ and the normal range was $0.3 \mathrm{mIU} / \mathrm{L}-3.6 \mathrm{mIU} / \mathrm{L}$. The intra-assay and inter-assay coefficient of variation of TSH was $0.7 \%-$ $1.9 \%$ and $1.6 \%-5.1 \%$, respectively. The measured concentration of $\mathrm{FT}_{4}$ was between $0.1 \mathrm{ng} / \mathrm{dL}$ and $10 \mathrm{ng} / \mathrm{dL}$ and the normal range was $0.8 \mathrm{ng} / \mathrm{dL}-1.7 \mathrm{ng} / \mathrm{dL}$. The intra-assay and inter-assay coefficient of variation was $1.1 \%-2.4 \%$ and $2.9 \%-4.8 \%$, respectively. With regard to $\mathrm{TPOAb}$, the measured concentration was between 1 $\mathrm{IU} / \mathrm{mL}$ and $2,000 \mathrm{IU} / \mathrm{mL}$ and the normal range was 1 $\mathrm{IU} / \mathrm{mL}-16 \mathrm{IU} / \mathrm{mL}$. The intra-assay and inter-assay coefficient of variation was $3.6 \%-7.4 \%$ and $7.9 \%-12.7 \%$, respectively.

According to guidelines for the diagnosis and treatment of pregnancy and postpartum thyroid diseases in China [19], we created independent reference values of thyroid hormones in our own laboratory. The $2.5^{\text {th }}-97.5^{\text {th }}$ percentile cut offs of TSH and $\mathrm{FT}_{4}$ were calculated in early pregnancy in TPOAb-negative women. It was $0.046 \mathrm{mIU} / \mathrm{L}-5.416 \mathrm{mIU} / \mathrm{L}$ for TSH and $0.716 \mathrm{ng} / \mathrm{dL}-$ $1.430 \mathrm{ng} / \mathrm{dL}$ for $\mathrm{FT}_{4}$. TPOAb $\geq 16 \mathrm{IU} / \mathrm{mL}$ was regarded as positive.

Isolated sub-clinical hypothyroidism was diagnosed as TSH greater than $5.416 \mathrm{mIU} / \mathrm{L}$ and $\mathrm{FT}_{4}$ between 0.716 
$\mathrm{ng} / \mathrm{dL}-1.430 \mathrm{ng} / \mathrm{dL}$, with negative TPOAb. Isolated hypothyroxinemia was regarded as TSH between 0.046 $\mathrm{mIU} / \mathrm{L}-5.416 \mathrm{mIU} / \mathrm{L}$ and $\mathrm{FT}_{4}$ less than $0.716 \mathrm{ng} / \mathrm{dL}$, with negative TPOAb. TSH between $0.046 \mathrm{mIU} / \mathrm{L}-5.416$ $\mathrm{mIU} / \mathrm{L}$ and $\mathrm{FT}_{4}$ between $0.716 \mathrm{ng} / \mathrm{dL}-1.430 \mathrm{ng} / \mathrm{dL}$ with positive TPOAb was defined as isolated positive TPOAb. Women with negative TPOAb, and TSH between $0.046 \mathrm{mIU} / \mathrm{L}-5.416 \mathrm{mIU} / \mathrm{L}$ and $\mathrm{FT}_{4}$ between $0.716 \mathrm{ng} / \mathrm{dL}-1.430 \mathrm{ng} / \mathrm{dL}$ were euthyroid.

\section{Definition of GDM}

GDM was screened on 24-28 gestational weeks. In the medical records, GDM was confirmed by standard diagnosis protocol in China by using a $100 \mathrm{~g}$ oral glucose tolerance test (OGTT). Fasting blood glucose, blood glucose at 1, 2, 3 hours after oral administration were assessed. The threshold was set as: (i) fasting blood glucose $\geq 5.3 \mathrm{mmol} / \mathrm{L}$, (ii) blood glucose at 1 hour after oral administration $\geq 10.0 \mathrm{mmol} / \mathrm{L}$, (iii) blood glucose at 2 hours after oral administration $\geq 8.6 \mathrm{mmol} / \mathrm{L}$, (iv) blood glucose at 3 hours after oral administration $\geq 7.8 \mathrm{mmol} / \mathrm{L}$. GDM was diagnosed when no less than two of the four parameters reached or exceeded the normal value [20].

\section{Data analysis}

Data analysis was performed using IBM SPSS Statistics 23.0. Descriptive statistics was used and presented as mean, standard deviation, and frequency. Independentsamples $t$ tests and one-way ANOVA were adopted to compare the differences among continuous variables. $\chi^{2}$ tests and Fisher's exact tests were used for categorical indicators. Logistic regression models were employed to assess the isolated impact of TSH, $\mathrm{FT}_{4}$ and TPOAb on GDM, and euthyroid women with negative TPOAb was regarded as the control group. In sensitive analysis, socio-economic and obstetric characteristics of women who were included and excluded were compared. Meanwhile, after excluding multiparas, overweight and obesity women prior to pregnancy and women with previous adverse pregnancy outcomes, the logistic regression models were re-run to observe whether the findings were materially changed. Statistical significance was set at the $\alpha=0.05$ level.

\section{Results}

With informed consents, a total of 3,585 completed questionnaires and serum samples were obtained, in which 2,023 women were in the $1^{\text {st }}$ trimester of pregnancy. After excluding samples with unclear information on gestational weeks and GDM, women who used ART for the index pregnancy, and women who had existing diabetes, hypertension and thyroid diseases, totally 1,779 samples with full data on thyroid function and GDM. Among these women, 96 had other kind of thyroid dysfunction, such as overt hypothyroidism, sub-clinical hypothyroidism with positive TPOAb, overt or subclinical hyperthyroidism, hypothyroxinemia with positive TPOAb or hypopituitarism (Fig. 1).

The overall prevalence of positive TPOAb was $15.3 \%$ $(309 / 2,023)$. The prevalence of GDM with isolated subclinical hypothyroidism, isolated hypothyroxinemia and isolated positive TPOAb was $2.9 \%(1 / 34), 2.8 \%(1 / 36)$ and $3.1 \%(7 / 224)$, respectively, all of which were higher than the rate $(1.2 \%, 17 / 1,389)$ found in the euthyroid women.

There were significant differences in maternal age and pre-pregnancy BMI among women in various thyroid functions. Women with isolated hypothyroxinemia and positive TPOAb were older than euthyroid women, and GDM women were older than non-GDM ones. Women with isolated hypothyroxinemia had higher pre-pregnancy BMI than women with isolated sub-clinical hypothyroidism, positive TPOAb and euthyroid women.

As far as TSH level was concerned, compared with euthyroid women, besides that it was higher in women with isolated sub-clinical hypothyroidism without doubt, women with positive TPOAb and hypothyroxinemia also had higher level of TSH (all $p$ values $<0.01$ ). As to $\mathrm{FT}_{4}$, compared with euthyroid women, besides those with hypothyroxinemia, women with sub-clinical hypothyroidism and positive TPOAb had lower level of $\mathrm{FT}_{4}$ (Table 1).

To investigate the impact of isolated TSH, $\mathrm{FT}_{4}$ and TPOAb on the likelihood of GDM, we incorporated logistic regression analysis. In model 1 , only various thyroid function was entered as the independent variable and euthyroidism was regarded as control. It showed that women with isolated positive TPOAb would more possibly suffered from GDM, RR and 95\%CI being 2.603 (1.067-6.351). In model 2, maternal age, education level, residence, pre-pregnancy BMI, previous adverse pregnancy outcomes, parity and pregnancy-specific stress were added as controlling variables. RR $(95 \% \mathrm{CI})$ for TPOAb was 2.541 (1.037-6.226). Despite that subjects were euthyroid, the reference range for $\mathrm{TSH}$ and $\mathrm{FT}_{4}$ levels were wide, and there would be still quite some variance in the euthyroid range. To truly investigate the independent effect of TPOAb, TSH and $\mathrm{FT}_{4}$ levels were further adjusted in model 3. The RR and $95 \% \mathrm{CI}$ for TPOAb didn't change after the two thyroid hormones were controlled (Table 2).

In sensitive analysis, we compared the socio-economic and obstetric characteristics in women who were included and excluded. It was found that the included women were younger and higher educated. More proportions of 


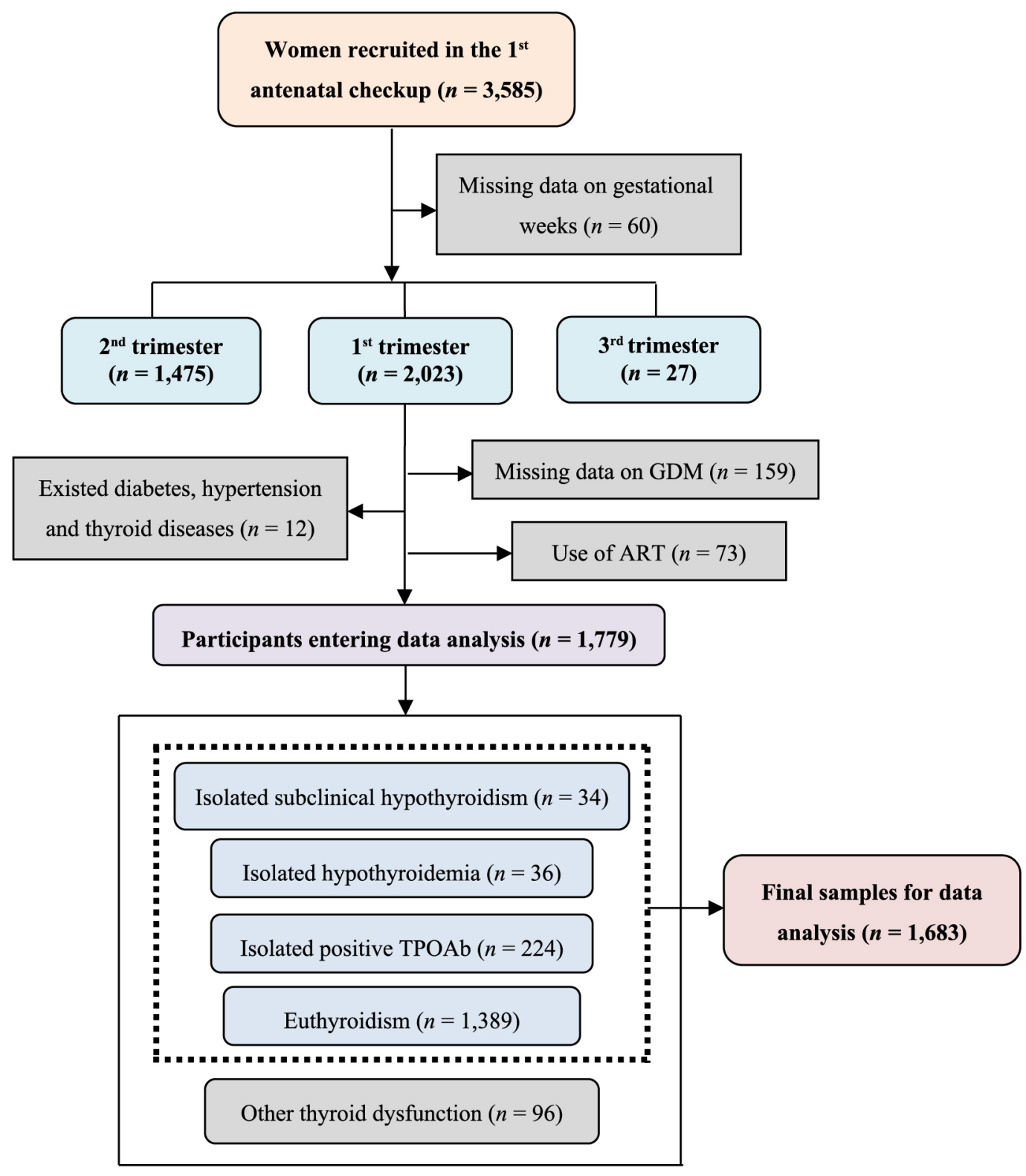

Fig. 1 Flow chart for participants recruitment

The figure had shown the process of participants recruitment in this study. With informed consents, a total of 3,585 completed questionnaires and serum samples were obtained, in which 2,023 women were in the $1^{\text {st }}$ trimester of pregnancy. After excluding samples with unclear information on gestational weeks and GDM, women who used ART for the index pregnancy, and women who had existing diabetes, hypertension and thyroid diseases, totally 1,779 samples with full data on thyroid function and GDM. Among these women, 96 had other kind of thyroid dysfunction, such as overt hypothyroidism and hyperthyroidism, subclinical hyperthyroidism, sub-clinical hypothyroidism with positive TPOAb and hypothyroxinemia with positive TPOAb and hypopituitarism. Finally 1,683 samples were included for formal data analysis.

Isolated sub-clinical hypothyroidism: TSH greater than $5.416 \mathrm{mIU} / \mathrm{L}, \mathrm{FT}_{4}$ between $0.716 \mathrm{ng} / \mathrm{dL}-1.430 \mathrm{ng} / \mathrm{dL}$, TPOAb negative; Isolated hypothyroxinemia: TSH between $0.046 \mathrm{mIU} / \mathrm{L}-5.416 \mathrm{mIU} / \mathrm{L}, \mathrm{FT}_{4}$ less than $0.716 \mathrm{ng} / \mathrm{dL}$, TPOAb negative; Isolated positive TPOAb: TSH between $0.046 \mathrm{mIU} / \mathrm{L}-5.416 \mathrm{mIU} / \mathrm{L}, \mathrm{FT}_{4}$ between $0.716 \mathrm{ng} / \mathrm{dL}-1.430 \mathrm{ng} / \mathrm{dL}$, TPOAb positive; Euthyroidism: TSH between $0.046 \mathrm{mIU} / \mathrm{L}-5.416 \mathrm{mIU} / \mathrm{L}, \mathrm{FT}_{4}$ between $0.716 \mathrm{ng} / \mathrm{dL}-1.430 \mathrm{ng} / \mathrm{dL}$, TPOAb negative;

Other thyroid dysfunction: including overt hypothyroidism and hyperthyroidism, subclinical hyperthyroidism, sub-clinical hypothyroidism with positive TPOA, hypothyroxinemia with positive TPOAb and hypopituitarism

included women were primiparas. No significant differences were found in $\mathrm{TSH}, \mathrm{FT}_{4}$ levels or the prevalence of positive TPOAb between the two groups (Table 3). And then, we respectively excluded women who were over- weight and obesity prior to pregnancy, women with previous adverse pregnancy outcome and multiparas, and re-ran the logistic regression models. In model 3, the associations between TPOAb and GDM were not materi- 
Table 1 Socio-economic and obstetric characteristics among different groups of pregnant women

\begin{tabular}{|c|c|c|c|c|c|c|}
\hline \multirow[b]{2}{*}{ Variables } & \multicolumn{4}{|c|}{ Grouping of women by thyroid function } & \multicolumn{2}{|c|}{$\begin{array}{l}\text { Grouping of women by } \\
\text { GDM status }\end{array}$} \\
\hline & $\begin{array}{l}\text { Isolated sub-clinical } \\
\text { hypothyroidism } \\
(n=34)\end{array}$ & $\begin{array}{c}\text { Isolated } \\
\text { hypothyroxinemia } \\
(n=36)\end{array}$ & $\begin{array}{l}\text { Isolated } \\
\text { positive } \\
\text { TPOAb } \\
(n=224)\end{array}$ & $\begin{array}{l}\text { Euthyroidism } \\
(n=1,389)\end{array}$ & $\begin{array}{c}\text { GDM } \\
(n=26)\end{array}$ & $\begin{array}{l}\text { non-GDM } \\
(n=1,657)\end{array}$ \\
\hline $\begin{array}{l}\text { Maternal age } \\
(\text { mean } \pm \mathrm{SD})\end{array}$ & $27.4 \pm 4.0$ & $28.2 \pm 3.4$ & $27.0 \pm 3.3$ & $26.7 \pm 3.2 *$ & $28.4 \pm 3.1$ & $26.8 \pm 3.2 *$ \\
\hline $\begin{array}{l}\text { Maternal educational } \\
\text { years }(\text { mean } \pm \mathrm{SD})\end{array}$ & $13.2 \pm 2.6$ & $14.3 \pm 2.5$ & $13.4 \pm 3.3$ & $13.3 \pm 3.2$ & $14.0 \pm 2.9$ & $13.3 \pm 3.2$ \\
\hline \multicolumn{7}{|l|}{$\begin{array}{l}\text { Monthly income per } \\
\text { capita (yuan) }(n / \%)^{\text {ab }}\end{array}$} \\
\hline$\leq 2,000$ & $18 / 52.9$ & $14 / 38.9$ & $127 / 56.7$ & $756 / 54.5$ & $12 / 46.2$ & $903 / 54.6$ \\
\hline $2,001-7,999$ & $15 / 44.1$ & $22 / 61.1$ & $97 / 43.3$ & $614 / 44.3$ & $14 / 53.8$ & $734 / 44.4$ \\
\hline$\geq 8,000$ & $1 / 2.9$ & $0 / 0$ & $0 / 0$ & $16 / 1.2$ & $0 / 0$ & $17 / 1.0$ \\
\hline \multicolumn{7}{|l|}{ Resident place $(n / \%)^{\mathrm{b}}$} \\
\hline Urban areas & $32 / 94.1$ & $34 / 94.4$ & $201 / 89.7$ & $1,214 / 87.6$ & $24 / 92.3$ & $1,457 / 88.1$ \\
\hline Rural areas & $2 / 5.9$ & $2 / 5.6$ & $23 / 10.3$ & $172 / 12.4$ & $2 / 7.7$ & $197 / 11.9$ \\
\hline $\begin{array}{l}\text { Pre-pregnant BMI } \\
(\text { mean } \pm \mathrm{SD})\end{array}$ & $20.5 \pm 3.0$ & $21.6 \pm 3.0$ & $20.4 \pm 2.0$ & $20.2 \pm 2.5 * *$ & $21.4 \pm 3.4$ & $20.2 \pm 2.5$ \\
\hline $\begin{array}{l}\text { Previous adverse } \\
\text { pregnancy outcome } \\
(n / \%)\end{array}$ & $20 / 58.8$ & $19 / 52.8$ & $103 / 46.0$ & $645 / 46.4$ & $16 / 61.5$ & $771 / 46.5$ \\
\hline Primiparity $(n / \%)$ & $33 / 97.1$ & $33 / 91.7$ & $215 / 96.0$ & $1,335 / 96.1$ & $24 / 92.3$ & $1,592 / 96.1$ \\
\hline $\begin{array}{l}\text { Pregnancy-specific } \\
\text { anxiety in } 1^{\text {st }} \text { trimester } \\
(\text { mean } \pm \mathrm{SD})\end{array}$ & $19.9 \pm 4.8$ & $19.5 \pm 5.0$ & $21.0 \pm 5.1$ & $20.9 \pm 5.0$ & $21.6 \pm 4.6$ & $20.9 \pm 5.0$ \\
\hline TSH (mIU/L) & $7.0 \pm 2.0$ & $2.2 \pm 1.1$ & $2.3 \pm 1.2$ & $1.8 \pm 1.1^{* *}$ & $1.9 \pm 1.7$ & $2.0 \pm 1.4$ \\
\hline $\mathrm{FT}_{4}(\mathrm{ng} / \mathrm{dL})$ & $0.93 \pm 0.13$ & $0.67 \pm 0.04$ & $0.97 \pm 0.15$ & $1.01 \pm 0.14^{* *}$ & $1.0 \pm 0.2$ & $1.0 \pm 0.2$ \\
\hline $\begin{array}{l}\text { Positive TPOAb } \\
(n / \%)\end{array}$ & - & - & - & - & $7 / 26.9$ & $217 / 13.1^{*}$ \\
\hline
\end{tabular}

a Fisher's exact tests; ${ }^{\mathrm{b}} 3$ missing data

$* p<0.05, * * p<0.01$

Table 2 Logistic regression models towards the impact of TSH, $\mathrm{FT}_{4}$ and TPOAb in early pregnancy on GDM [RR(95\%CI)]

\begin{tabular}{lccc}
\hline & Model 1 & Model 2 & Model 3 \\
\hline Euthyroidism & 1.00 & 1.00 & 1.00 \\
Isolated sub-clinical hypothyroidism & $2.446(0.316-18.924)$ & $1.920(0.237-15.524)$ & $1.920(0.237-15.524)$ \\
Isolated hypothyroxinemia & $2.306(0.298-17.814)$ & $1.609(0.203-12.785)$ & $1.609(0.203-12.785)$ \\
Isolated positive TPOAb & $2.603(1.067-6.351)$ & $2.541(1.037-6.226)$ & $2.541(1.037-6.226)$ \\
\hline
\end{tabular}

Model 1: various thyroid function was entered as the independent variable;

Model 2: Model 1 + maternal age, maternal education level, residence, pre-pregnancy BMI, previous adverse pregnancy outcomes, parity and pregnancy-specific stress;

Model 3: Model $2+\mathrm{TSH}$ and $\mathrm{FT}_{4}$ in early pregnancy 
Table 3 Socio-economic and obstetric characteristics between women included and excluded

\begin{tabular}{|c|c|c|}
\hline Variables & $\begin{array}{l}\text { Included women } \\
\quad(n=1,779)\end{array}$ & $\begin{array}{l}\text { Excluded women } \\
\quad(n=244)\end{array}$ \\
\hline Maternal age $($ mean $\pm \mathrm{SD}) * *$ & $26.8 \pm 3.2$ & $27.6 \pm 4.2$ \\
\hline Maternal educational years $($ mean $\pm \mathrm{SD}) * *$ & $13.3 \pm 3.2$ & $12.3 \pm 3.5$ \\
\hline \multicolumn{3}{|l|}{ Monthly income per capita (yuan) $(n / \%)^{*}$} \\
\hline$\leq 2,000$ & $965 / 54.3$ & $141 / 57.8$ \\
\hline $2,001-7,999$ & $792 / 44.6$ & $96 / 39.3$ \\
\hline$\geq 8,000$ & $19 / 1.1$ & $7 / 2.9$ \\
\hline \multicolumn{3}{|l|}{ Resident place $(n / \%)$} \\
\hline Urban areas & $1,567 / 88.2$ & $205 / 84.0$ \\
\hline Rural areas & $209 / 11.8$ & $39 / 16.0$ \\
\hline Pre-pregnant $\mathrm{BMI}($ mean $\pm \mathrm{SD})$ & $20.2 \pm 2.5$ & $20.4 \pm 2.6$ \\
\hline Previous adverse pregnancy outcome $(n / \%)$ & $837 / 47.0$ & $129 / 52.9$ \\
\hline Primiparity $(n / \%)^{* *}$ & $1,708 / 96.0$ & $222 / 91.0$ \\
\hline Pregnancy-specific anxiety in $1^{\text {st }}$ trimester $($ mean $\pm S D)$ & $20.9 \pm 5.0$ & $21.3 \pm 5.6$ \\
\hline TSH (mIU/L) & $2.0 \pm 1.9$ & $2.1 \pm 1.6$ \\
\hline $\mathrm{FT}_{4}(\mathrm{ng} / \mathrm{dL})$ & $1.0 \pm 0.2$ & $1.0 \pm 0.2$ \\
\hline Positive TPOAb $(n / \%)$ & $262 / 14.7$ & $47 / 19.3$ \\
\hline
\end{tabular}

ally changed, RR (95\%CI) being 3.101 (1.237-7.775), 2.769 (1.125-6.811) and 2.576 (1.792-8.372), respectively.

\section{Discussion}

Based on a prospective cohort study design, we found that isolated positive TPOAb with normal thyroid function in early pregnancy independently predicted GDM.

The prevalence of GDM in this study is relatively low. The diagnostic criteria of GDM had been reformulated for several times in China. During the period of our study, we had used the criteria developed by obstetric group of Obstetrics and Gynecology Branch and GDM group in Perinatal Medicine Branch in Chinese Medical Association in 2007. In 2014, in accordance with the new GDM diagnostic criteria announced by the American Diabetes Association (ADA), the two groups modified the criteria and issued the guidelines for GDM diagnosis and treatment (2014) [21]. Because we don't have the raw data on blood glucose, we can not exactly describe the GDM rate detected by the adoption the new criteria. But the increasing trend in GDM prevalence needs to be closely noticed.

$\mathrm{TPOAb}$ is an important indicative of thyroid inflammation, and can be detected in $10 \%-20 \%$ of reproductive women [22]. In the current study, the overall detection rate of positive TPOAb in pregnant women was $15.3 \%$. Positive TPOAb is a pathological change that indicates the activation of thyroid autoimmune process, and it is a common cause of hypothyroidism in women of childbearing age [23]. Thyroid autoimmunity is the potential risk factor of diabetes mellitus. It is estimated that GDM is caused by $\beta$-cell dysfunction in most patients. The underlying mechanism is increased insulin resistance rather than decrease in insulin secretion [24]. Researchers demonstrated that the presence of TPOAb but not thyroglobulin antibodies ( $\mathrm{TgAb})$ was associated with a higher risk of GDM [9]. Ying $\mathrm{H}$ and colleagues found that isolated positive TPOAb in early pregnancy increased the risk of GDM [25], and the rate of positive TPOAb was higher in pregnant women with GDM than those without GDM [10, 26], which was in accordance with our findings. The link between thyroid autoimmune disorder and insulin resistance could be the inflammatory events associated with both conditions. For example, serum cytokine levels are increased both in patients with thyroid autoimmunity and insulin resistance [27, 28]. Presence of inflammatory markers is related to diabetes mellitus in pregnant women [29, 30], and thyroid dysfunction has also been related to insulin resistance [31, 32]. TPOAb might serve as a marker for an inflammatory process that links thyroid autoimmunity with insulin resistance, and positive TPOAb makes pregnant women 
susceptible to GDM. Other studies, however, have found inconsistent conclusions. Pascual Corrales E et al. observed an association between higher prevalence of TPOAb and higher GDM rate, but no statistical significance was identified due to the small sample size [24]. Some other investigations didn't find the relationship between thyroid autoimmunity during pregnancy and GDM [5, 14, 33-36].

It is worth noting that the majority of women with positive thyroid autoantibodies are euthyroid [23]. In this study, although TPOAb-positive women had higher TSH and lower $\mathrm{FT}_{4}$ levels than women with TPOAb-negative pregnant women with normal thyroid function, isolated positive TPOAb was still associated with GDM after controlling for maternal $\mathrm{TSH}$ and $\mathrm{FT}_{4}$ levels in early pregnancy. The predictive effect of TPOAb on GDM may independent of $\mathrm{TSH}$ or $\mathrm{FT}_{4}$.

This study focused on TPOAb-negative subclinical hypothyroidism and TPOAb-negative hypothyroxinemia. No association was found between these two mild thyroid dysfunction and GDM in early pregnancy. There was no significant difference in TSH or $\mathrm{FT}_{4}$ levels between GDM and non-GDM pregnant women. The relationship between sub-clinical hypothyroidism and GDM is controversial. Many studies had identified that there was no significant difference in $\mathrm{TSH}, \mathrm{FT}_{4}$ or TPOAb levels between GDM and non-GDM pregnant women [14, 37], and no correlation between sub-clinical hypothyroidism and GDM was indicated [8, 33, 38]. Meanwhile, Toulis KA reported that sub-clinical hypothyroidism was related to the occurrence of GDM [39]. Ying $\mathrm{H}$ et al. [25] proved that sub-clinical hypothyroidism (regardless of TPOAb status) in early pregnancy was associated with an increased risk of GDM. Sub-clinical hypothyroidism of a non-autoimmune origin may not be regarded as markers for other autoimmune conditions that might, in turn, be responsible for the GDM, and may not attribute to increased inflammatory factors, such as C-reactive protein [40]. Furthermore, our finding was different from two other studies in China [10,11], which demonstrated significant difference in $\mathrm{FT}_{4}$ levels between GDM and non-GDM women. It may also support a hypothesis that hypothyroidism during pregnancy might affect glucose metabolism only if the condition was associated with antibodies [5], and an underlying enhanced global autoimmune state is relevant to GDM. In the current study, sub-clinical hypothyroidism and hypothyroidism women with positive TPOAb were brought into analysis and the prevalence of GDM was $7.1 \%$ and $0 \%$ respectively. Compared with sub-clinical hypothyroidism and hypothyroidism women with negative TPOAb respectively, no statistical significance was identified. Further studies with measurements of specific biomark- ers related to autoimmunity (glutamic acid decarboxylase, islet cell, or insulin antibodies) during pregnancy could investigate whether the association between TPOAb and GDM is the consequence of a widespread autoimmune disorder $[9,41]$.

This study demonstrated an independent predictive effect of isolated positive TPOAb and GDM. Since it was based on a population-based prospective cohort, there exited reasonable chronological order when explaining the causality. In other words, positive TPOAb was tested prior to GDM diagnosis, and predicted the occurrence of GDM. Based on ethical considerations, we just collected maternal serum samples, diagnosed GDM cases and abstracted pregnancy outcomes in sequence. It was a natural process of observation. No thyroid function assays were carried by using the serum samples during pregnancy, and thus no additional intervention were administrated to the participants. After data collection was complemented, thyroid function in early pregnancy was then retrospectively detected and its impact on the subsequent GDM was then discussed. These processes were described in detail in the informed consents. Secondly, due to the racial and geographical differences between TSH and $\mathrm{FT}_{4}$, this study adopted a pregnancyspecific thyroid hormone reference suitable for the study population, and strictly defined the threshold when TPOAb was negative, so as to accurately classify women's thyroid function. In the third, isolated subclinical hypothyroidism, hypothyroxinemia with negative TPOAb, and isolated positive TPOAb with normal thyroid function were separated out in this study. We attempted to eliminate the effects of mixed thyroid dysfunction and autoimmune antibodies on glucose metabolism and highlight the independent predictive roles of TSH, $\mathrm{FT}_{4}$ and TPOAb in GDM occurrence. Previous studies had revealed significant association between thyroid autoantibodies in euthyroid women with miscarriage and premature delivery $[19,42]$. The predictive effect of isolated positive TPOAb on GDM, independent of TSH or $\mathrm{FT}_{4}$, has provided evidence for the impact of "mild" thyroid dysfunction in early pregnancy on adverse maternal health.

There are also several limitations in the present study that deserve acknowledgment. Pregnant women may require additional iodine intake to avoid iodine deficiency disorders. In this study, we did not have available data on possible dietary iodine supplementation, nor did we measure urinary iodine excretion to test for individual iodine status. Secondly, it is possible that women who are euthyroid with positive TPOAb in early pregnancy carry a significant and progressive risk of becoming hypothyroid during gestation. We did not collect the serum samples during the $2^{\text {nd }}$ and $3^{\text {rd }}$ trimester of preg- 
nancy, thus we could not assess what changes occurred in the thyroid function of these women and the relationship with later glucose metabolism. In the third, $\mathrm{TgAb}$ is another important indicator and inflammatory marker of thyroid autoimmunity. Although no association between $\mathrm{TgAb}$ and GDM had been reported in some previous studies $[9,36]$, the combined effect of TgAb and TPOAb in predicting GDM needs to be evaluated in further studies.

\section{Conclusions}

Isolated positive TPOAb in early pregnancy in euthyroid women is associated with an increased risk of GDM. The effect was independent of TSH and $\mathrm{FT}_{4}$ levels. Thyroid autoimmunity in early pregnancy, rather than isolated $\mathrm{TSH}_{\mathrm{Sr}} \mathrm{FT}_{4}$, may predict the occurrence of GDM.

\section{Acknowledgement}

This study is funded by National Natural Science Foundation of China (81872630, 30901202, 81330068 and 81472991$)$. It is also supported by Project of Academic and Technical Leaders of Anhui Province (2017H140), Excellent Young Talents Fund Program of Higher Education Institutions of Anhui Province (2014), Prominent Talents Program of Anhui Medical University (2014). The authors would like to thank all the participants, field workers and master students for their valuable contributions to this research.

\section{Competing Financial Interests Statement}

The authors declare no conflict of competing financial interest.

\section{Reference}

1. American Diabetes Association (2011) Diagnosis and classification of diabetes mellitus. Diabetes Care 34 Suppl 1: S62-S69.

2. Zhang SL (2008) Diabetes mellitus. In: Le J (ed) Obstetrics and Gynecology (7th). People's Medical Publishing House, Beijing, China: 150-154 (In Chinese).

3. Bellamy L, Casas JP, Hingorani AD, Williams D (2009) Type 2 diabetes mellitus after gestational diabetes: a systematic review and meta-analysis. Lancet 373: 1773 1779 .

4. Lambadiari V, Mitrou P, Maratou E, Raptis AE, Tountas $\mathrm{N}$, et al. (2011) Thyroid hormones are positively associated with insulin resistance early in the development of type 2 diabetes. Endocrine 39: 28-32.

5. Männistö T, Vääräsmäki M, Pouta $A$, Hartikainen $A L$, Ruokonen A, et al. (2010) Thyroid dysfunction and autoantibodies during pregnancy as predictive factors of pregnancy complications and maternal morbidity in later life. $J$ Clin Endocrinol Metab 95: 1084-1094.

6. Casey BM, Dashe JS, Wells CE, McIntire DD, Byrd W, et al. (2005) Subclinical hypothyroidism and pregnancy outcomes. Obstet Gynecol 105: 239-245.

7. Olivieri A, Valensise H, Magnani F, Medda E, De Angelis S, et al. (2000) High frequency of antithyroid autoantibodies in pregnant women at increased risk of gestational diabetes mellitus. Eur J Endocrinol 143: 741-747.

8. Tudela CM, Casey BM, McIntire DD, Cunningham FG (2012) Relationship of subclinical thyroid disease to the incidence of gestational diabetes. Obstet Gynecol 119: 983-988.

9. Karakosta P, Alegakis D, Georgiou V, Roumeliotaki T, Fthenou E, et al. (2012) Thyroid dysfunction and autoantibodies in early pregnancy are associated with increased risk of gestational diabetes and adverse birth outcomes. $J$
Clin Endocrinol Metab 97: 4464-4472.

10. Lin N, Liu H (2016) Analysis of relationship between gestational daibetes mellitus and thyroid autoimmunity. China Prac Med 11: 41-42 (In Chinese).

11. Xu J (2017) Correlation analysis between urinary iodine, TPOAB, FT4, TSH and gestational diabetes mellitus during pregnancy. Chin J Mod Drug Appl 11: 12-14 (In Chinese).

12. Shahbazian H, Shahbazian N, Rahimi Baniani M, Yazdanpanah L, Latifi SM (2013) Evaluation of thyroid dysfunction in pregnant women with gestational and pregestational diabetes. Pak J Med Sci 29: 638-641.

13. Ruf J, Carayon P (2006) Structural and functional aspects of thyroid peroxidase. Arch Biochem Biophys 445: 269277.

14. Agarwal MM, Dhatt GS, Punnose J, Bishawi B, Zayed R (2006) Thyroid function abnormalities and antithyroid antibody prevalence in pregnant women at high risk for gestational diabetes mellitus. Gynecol Endocrinol 22: 261-266.

15. Tao FB, Hao JH, Huang K, Su PY, Cheng DJ, et al. (2013) Cohort profile: the China-Anhui birth cohort study. Int J Epidemiol 42: 709-721.

16. Xiao L, Tao FB, Zhang J, Hao J, Xu S, et al. (2012) Development and reliability evaluation of a pregnancyrelated anxiety questionnaire. Chin J Public Health 28: 275-277 (In Chinese).

17. Zhang J, Hao J, Tao F, Wang H, Zhu P, et al. (2011) Influencing factors of pregnancy-related anxiety in first trimester of pregnancy. Chin J Public Health 27: 969-971 (In Chinese).

18. Zhou B, Coorperative Meta-Analysis Group Of Working Group On Obesity In China (2002) Prospective study for cut-off points of body mass index in Chinese adults. 
Zhonghua Liu Xing Bing Xue Za Zhi 23: 431-434 (In Chinese).

19. Endocrinology Branch of Chinese Medical Association, Perinatal Medicine Branch in Chinese Medical Association (2012) Guidelines for diagnosis and treatment of thyroid diseases during pregnancy and after childbirth. Chin J Endocrinol Metab 28: 354-371 (In Chinese).

20. Obstetric group of Obstetrics and Gynecology Branch in Chinese Medical Association, GDM group of Perinatal Medicine Branch in Chinese Medical Association (2007) Recommended guidelines for clinical diagnosis and treatment of gestational diabetes (draft). Chin J Obstet Gynecol 42: 426-428 (In Chinese).

21. Obstetric group of Obstetrics and Gynecology Branch in Chinese Medical Association, GDM group of Perinatal Medicine Branch in Chinese Medical Association (2014) Guidelines for diagnosis and treatment of gestational diabetes (2014). Chin J Obstet Gynecol 8: 561-568 (In Chinese).

22. Prummel MF, Wiersinga WM (2005) Thyroid peroxidase autoantibodies in euthyroid subjects. Best Pract Res Clin Endocrinol Metab 19: 1-15.

23. Stagnaro-Green A, Pearce E (2012) Thyroid disorders in pregnancy. Nat Rev Endocrinol 8: 650-658.

24. Pascual Corrales E, Andrada P, Aubá M, Ruiz Zambrana A, Guillén Grima F, et al. (2014) Is autoimmune thyroid dysfunction a risk factor for gestational diabetes? Endocrinol Nutr 61: 377-381 (In Spanish).

25. Ying H, Tang YP, Bao YR, Su XJ, Cai X, et al. (2016) Maternal TSH level and TPOAb status in early pregnancy and their relationship to the risk of gestational diabetes mellitus. Endocrine 54: 742-750.

26. Zhou J, Gao W, Zhang W (2016) Association between thyroid antibody and glycated hemoglobin in GDM patients with normal thyroid function. Hebei Medicine 22: 2038-2040 (In Chinese).

27. Drugarin D, Negru S, Koreck A, Zosin I, Cristea C (2000) The pattern of a $\mathrm{T}(\mathrm{H}) 1$ cytokine in autoimmune thyroiditis. Immunol Lett 71: 73-77.

28. Winer DA, Winer S, Chng MH, Shen L, Engleman EG (2014) B lymphocytes in obesity-related adipose tissue inflammation and insulin resistance. Cell Mol Life Sci 71: 1033-1043.

29. Qiu C, Sorensen TK, Luthy DA, Williams MA (2004) A prospectivestudy of maternal serum C-reactive protein (CRP) concentrations and risk of gestational diabetes mellitus. Paediatr Perinat Epidemiol 18: 377-384.

30. Wolf M, Sandler L, Hsu K, Vossen-Smirnakis K, Ecker JL, et al. (2003) First-trimester C-reactive protein and sub- sequent gestational diabetes. Diabetes Care 26: 819-824.

31. Galofré JC, Pujante P, Abreu C, Santos S, Guillen-Grima F, et al. (2008) Relationship between thyroidstimulatinghormone and insulin in euthyroid obese men. Ann Nutr Metab 53: 188-194.

32. Maratou E, Hadjidakis DJ, Kollias A, Tsegka K, Peppa M, et al. (2009) Studies of insulin resistance in patients with clinical and subclinical hypothyroidism. Eur J Endocrinol 160: 785-790.

33. Cleary-Goldman J, Malone FD, Lambert-Messerlian G, Sullivan L, Canick J, et al. (2008) Maternal thyroid hypofunction and pregnancy outcome. Obstet Gynecol 112: $85-92$.

34. Vitacolonna E, Lapolla A, Di Nenno B, Passante A, Bucci I, et al. (2012) Gestational diabetes and thyroid autoimmunity. Int J Endocrinol 2012: 867415.

35. Montaner P, Juan L, Campos R, Gil L, Corcoy R (2008) Is thyroid autoimmunity associated with gestational diabetes mellitus? Metabolism 57: 522-525.

36. Chen LM, Zhang Q, Si GX, Chen QS, Ye EL, et al. (2015) Associations between thyroid autoantibody status and abnormal pregnancy outcomes in euthyroid women. Endocrine 48: 924-928.

37. Ortega-González C, Liao-Lo A, Ramírez-Peredo J, Cariño N, Lira J, et al. (2000) Thyroid peroxidase antibodies in Mexican-born healthy pregnant women, in women with type 2 or gestational diabetes mellitus, and in their offspring. Endocr Pract 6: 244-248.

38. Chen LM, Du WJ, Dai J, Zhang Q, Si GX, et al. (2014) Effects of subclinical hypothyroidism on maternal and perinatal outcomes during pregnancy: a single-center cohort study of a Chinese population. PLoS One 9: e109364.

39. Toulis KA, Stagnaro-Green A, Negro R (2014) Maternal subclinical hypothyroidsm and gestational diabetes mellitus: a meta-analysis. Endocr Pract 20: 703-714.

40. Christ-Crain M, Meier C, Guglielmetti M, Huber PR, Riesen W, et al. (2003) Elevated C-reactive protein and homocysteine values: cardiovascular risk factors in hypothyroidism? A cross-sectional and a double-blind, placebo-controlled trial. Atherosclerosis 166: 379-386.

41. Nilsson C, Ursing D, Törn C, Aberg A, Landin-Olsson M (2007) Presence of GAD antibodies during gestational diabetes mellitus predicts type 1 diabetes. Diabetes Care 30: 1968-1971.

42. Thangaratinam S, Tan A, Knox E, Kilby MD, Franklyn J, et al. (2011) Association between thyroid autoantibodies and miscarriage and preterm birth: meta-analysis of evidence. BMJ 342: d2616. 